\title{
Raman imaging of doping domains in graphene on $\mathrm{SiO}_{2}$
}

\author{
C. Stampfer, ${ }^{a)}$ F. Molitor, D. Graf, and K. Ensslin \\ Solid State Physics Laboratory, ETH Zurich, 8093 Zurich, Switzerland
}

\begin{abstract}
A. Jungen and C. Hierold
Micro and Nanosystems, ETH Zurich, 8092 Zurich, Switzerland

L. Wirtz

Institute for Electronics, Microelectronics, and Nanotechnology (CNRS UMR-8520), Department ISEN, B.P. 60069 Villeneuve d'Ascq Cedex and European Theoretical Spectroscopy Facility (ETSF), E-20018 San Sebastián, Spain
\end{abstract}

(Received 26 September 2007; accepted 1 November 2007; published online 12 December 2007)

\begin{abstract}
We present spatially resolved Raman images of the $G$ and 2D lines of single-layer graphene flakes. The spatial fluctuations of $\mathrm{G}$ and 2D lines are correlated and are thus shown to be affiliated with local doping domains. We investigate the position of the 2D line-the most significant Raman peak to identify single-layer graphene-as a function of charging up to $|n| \approx 4 \times 10^{12} \mathrm{~cm}^{-2}$. Contrary to the $\mathrm{G}$ line which exhibits a strong and symmetric stiffening with respect to electron and hole doping, the 2D line shows a weak and slightly asymmetric stiffening for low doping. Additionally, the linewidth of the 2D line is, in contrast to the $\mathrm{G}$ line, doping independent making this quantity a reliable measure for identifying single-layer graphene. (C) 2007 American Institute of Physics. [DOI: 10.1063/1.2816262]
\end{abstract}

Graphene has attracted increasing attention over the las
few years. ${ }^{1,2}$ Its unique electronic properties, ${ }^{3,4}$ mainly due to
the linear energy versus momentum dispersion and the elec
tron hole symmetry near the charge neutrality point, make $\mathrm{i}$
an interesting nanomaterial for high mobility electronics. Raman spectroscopy has proven to be a powerful tool to distinguish single-layer graphene from few-layer graphene and graphite. ${ }^{7-11}$ The particular electronic structure of graphite and graphene leads to Kohn anomalies in the phonon dispersion at the $\Gamma$ and $K$ points. ${ }^{12-14}$ Additionally, the BornOppenheimer approximation, which is usually employed for the calculation of phonon frequencies, is no longer valid because the vibration period is much smaller than the electronmomentum relaxation time. Furthermore, a pronounced stiffening of the Raman $G$ line upon positive or negative charging ( $p$ or $n$ doping) of the graphene sheet is observed. ${ }^{15-17}$ In this paper, we investigate how spatially resolved Raman spectroscopy can be used to probe doping domains and local charge fluctuations. While electron-hole puddles (i.e., local charge fluctuations) have been predicted to be responsible for the finite conductance at vanishing (average) charge carrier density ${ }^{18}$ and have recently been observed using a scanning single electron transistor, ${ }^{19}$ the identification of different doping domains might be desirable to investigate graphene devices. Here, we report on Raman measurements on back gate controlled charged graphene and on Raman imaging of doping fluctuations of isolated graphene flakes. We focus on the correlation between the shifts of the $\mathrm{G}$ line and $2 \mathrm{D}$ line (or $\mathrm{D}^{*}$ line). The latter one is the most significant Raman peak in single-layer graphene. ${ }^{7,9}$ Within the low charging regime (up to $\pm 4 \times 10^{12} \mathrm{~cm}^{-2}$ ) obtained in our experiments, the 2D line stiffens for both electron and hole charging while its linewidth (in contrast to the $\mathrm{G}$ line) is not affected by charging. A good correlation be-

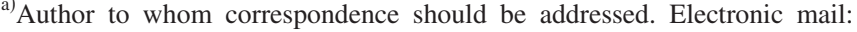
stampfer@phys.ethz.ch tween the shift of $\mathrm{G}$ and 2D lines is observed. However, the spectral resolution and lateral resolution are not sufficient to resolve electron-hole puddles, as shown in Ref. 19. Therefore, we refer to charging (i.e., doping) domains rather than to local electron-hole puddles.

We present both Raman images of isolated graphene and Raman measurements on electrically contacted single-layer graphene on $300 \mathrm{~nm} \mathrm{SiO}_{2}$, where highly doped $\mathrm{Si}$ is used as back gate. The samples are prepared by micromechanical cleavage $^{20}$ and Raman imaging ${ }^{21}$ is used to select singlelayer graphene flakes. ${ }^{7,9}$ By electron-beam lithography, we pattern scanning force microscopy premapped electrodes $(5 \mathrm{~nm} \mathrm{Cr} / 60 \mathrm{~nm} \mathrm{Au})$ on the graphene flakes, which finally allows us to apply a back gate voltage $V_{g}$ between the $\mathrm{Si}^{++}$ substrate and graphene. The experimental setup is shown in Fig. 1(a). The Raman data are recorded by using a laser excitation of $532 \mathrm{~nm}\left(E_{L}=2.33 \mathrm{eV}\right)$ through a single-mode optical fiber, whose spot size is limited by diffraction. A long working distance focusing lens with numerical aperture of approximately 0.80 is used to obtain a spot size of approximately $400 \mathrm{~nm}$. We use a laser power below $2 \mathrm{~mW}$ such that heating effects can be neglected. ${ }^{22}$ A Raman image of a measured device is shown in Fig. 1(b) and the Raman spectra corresponding to point $\mathrm{P}$ [Fig. 1(b)] for a charged and charge-neutral case are plotted in Fig. 1(c). The $\mathrm{G}$ line shifts by approximately $3 \mathrm{~cm}^{-1}$ due to hole charging (of $n \approx-4$ $\times 10^{12} \mathrm{~cm}^{-2}$ ) and a corresponding (hardly visible) small shift in the $2 \mathrm{D}$ line is observed too. Figure 2 shows Raman data for varying back gate voltages $V_{g}$ [Figs. 2(a) and 2(b)], which by utilizing a simple capacitor model can be substituted by the electron/hole concentration $n=\alpha\left(V_{g}-V_{g}^{D}\right)$. Here, $\alpha \approx 7.2 \times 10^{10} \mathrm{~cm}^{-2} / \mathrm{V}$ (Ref. 3) and $V_{g}^{D} \approx 2.5 \mathrm{~V}$ mark the charge neutrality point, which has been determined by transport measurements. A typical back gate characteristic of the investigated device is shown in Fig. 4 in Ref. 23. The symmetric hole and electron charging-dependent stiffening of the $E_{2 g} \Gamma$ phonon was recently explained as the effect of 
(a)
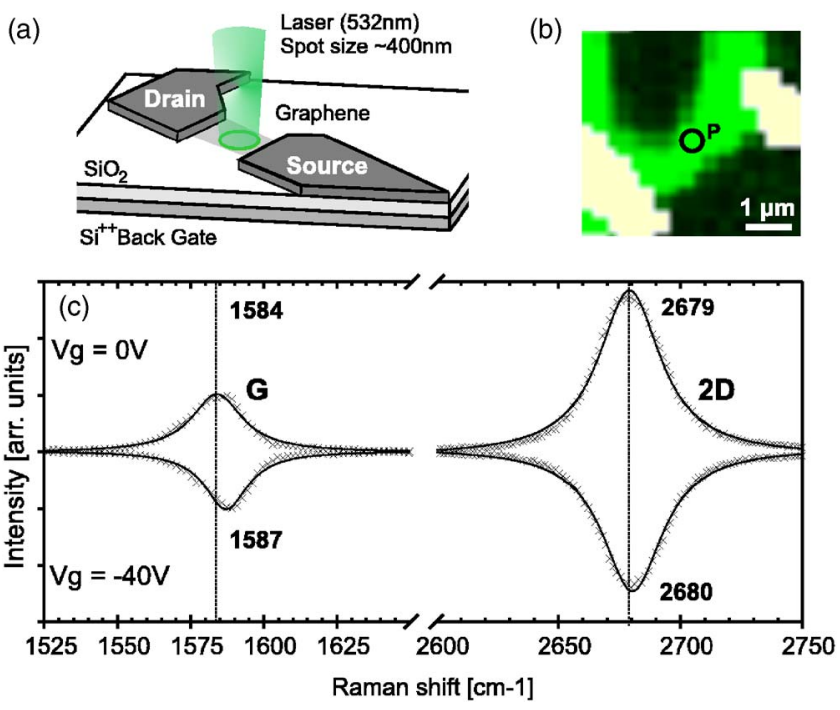

FIG. 1. (Color online) (a) Schematic illustration of a graphene sample with incident laser. (b) Raman image of a contacted single-layer graphene flake, where the integrated 2D line (green; dark) and the inverse Si line (at approximately $520 \mathrm{~cm}^{-1}$ ), which is screened by metal electrodes (yellow; bright), is colored. (c) Raman spectra of the G and 2D lines of chargeneutral and charged graphene measured at spot $\mathrm{P}$ marked in (b) at $T$ $=295 \mathrm{~K}$.

nonadiabaticity, ${ }^{14-17}$ i.e., the fact that the time scale of phonon oscillations in graphite is not long compared to the electron relaxation time [see, e.g., Eq. (6) in Ref. 16, and dashed line in Fig. 2(c)]. Results of time-dependent perturbation theory were, however, so far only presented for phonons at the $\Gamma$ point. For the phonon between $K$ and $M$ which is responsible for the $2 \mathrm{D}$ line, it has been argued ${ }^{24}$ that nonadiabatic effects are negligible and the influence of charging can be reproduced by a standard adiabatic phonon calculation. While nonadiabatic calculations predict a phonon stiffening for hole doping and a phonon softening for electron doping, ${ }^{24,25}$ we observe [see Fig. 2(d)] a slight (asymmetric) stiffening for both electron and hole dopings in agreement with the measurements of Ref. 17.

Another significant difference between the $G$ and 2D lines is their linewidth [full width at half maximum (FWHM) shown in Fig. 2(e)]. The $\mathrm{G}$ phonon $\left(q_{\mathrm{G}} \approx 0\right)$ shows a rather strong change as function of carrier density ${ }^{16,17}$ which is due to the fact that the Pauli exclusion principle prevents the phonon from decaying into an electron-hole pair for $\left|E_{F}\right|$ $>\hbar \omega_{G} / 2$, as illustrated in Fig. 2(f). ${ }^{17}$ The decay of the dispersive $\mathrm{D}$ phonon with large wave vector $q_{\mathrm{D}}$ is unaffected by the Pauli exclusion principle for low doping [Fig. 2(g)]. It is expected that the 2D linewidth stays constant up to a charging that corresponds to a Fermi level shift as large as the exciting laser energy $\left|E_{F}\right| \approx E_{L}$. This has a practical implication: since the peak width of the $2 \mathrm{D}$ line, which has been recognized as the most striking feature to distinguish singlelayer from few-layer graphene, ${ }^{7-9}$ is insensitive to doping, it is a reliable-doping-independent measure for identifying single-layer graphene.

Spatially resolved Raman spectroscopy (Raman imaging) sensing the doping-dependent $\mathrm{G}$ and $2 \mathrm{D}$ line shifts provides an interesting tool to investigate charge fluctuations and doping domains in graphene. Here, we present an example of Raman images $(80 \times 45$ pixels $)$ of a graphene flake on $\mathrm{SiO}_{2}$ (Fig. 3), where most attention has been paid to the $\mathrm{G}$ Downloaded 27 Aug 2008 to 129.132.208.18. Redistribution subje
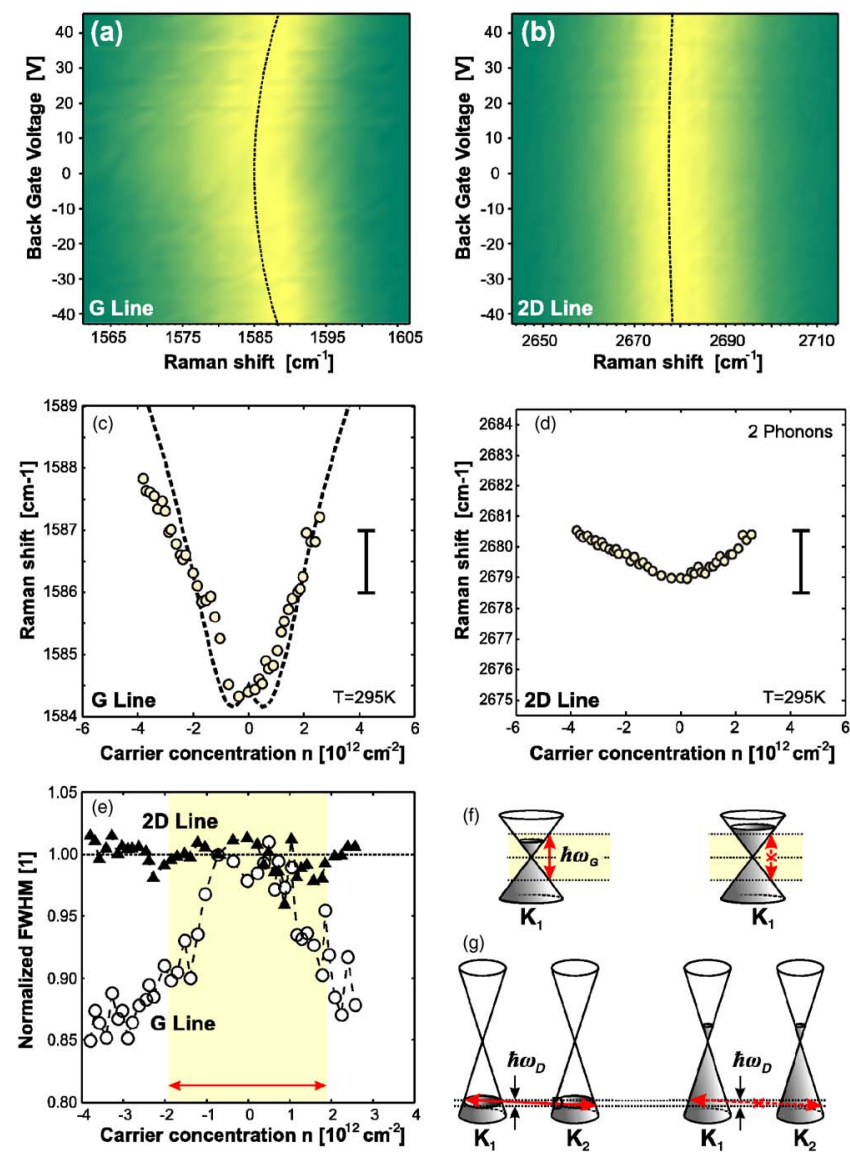

FIG. 2. (Color online) Raman spectroscopy of the $G$ and 2D lines of charged graphene. (a) and (b) show two-dimensional maps of the $\mathrm{G}$ and $2 \mathrm{D}$ lines, respectively, as function of applied back gate voltages. Both plots are peak height normalized. (c) shows the G line peak shift [extracted from (a) as function of the induced carrier concentration. Dashed lines show theoretical expectations for finite temperature (Ref. 14)]. (d) Same for the 2D peak shift. The same relative frequency scale as in (c) is used, indicated by the horizontal scale bars $\left(1 \mathrm{~cm}^{-1} /\right.$ phonon). (e) shows the normalized bandwidth (FWHM) of G and 2D as function of doping. (f) Schematic illustration of the presence (left panel) and absence (right panel) of a $\Gamma$ phonon decay into an electron-hole pair. $(\mathrm{g})$ The presence and absence of D-phonon decay into an electron-hole pair. $K_{1}$ and $K_{2}$ denote the two inequivalent $K$ points.

and 2D peak positions and widths, their fluctuations and cross correlations. The average G peak position [Fig. 3(c)] $\bar{\omega}_{\mathrm{G}}$ measured on the flake shown in Fig. 3(a), is $1585.4 \mathrm{~cm}^{-1}$ and the root mean square of the peak fluctuations is $3.3 \mathrm{~cm}^{-1}$. This fluctuation also nicely explains the wide spread range of $\bar{\omega}_{\mathrm{G}}$ reported in the literature. ${ }^{7-11}$ The 2D line [Fig. 3(d)] is centered around $\bar{\omega}_{2 \mathrm{D}}=2679.4 \mathrm{~cm}^{-1}$ and fluctuates with $0.9 \mathrm{~cm}^{-1}$. The ratio of the fluctuations of the $\mathrm{G}$ and 2D line agrees well with the ratio of the doping-dependent $G$ and 2D stiffening [Figs. 2(c) and 2(d)] of $\approx 3.2$. In Figs. 3(c) and 3(d), doped regions on the imaged flake can be observed. ${ }^{11}$ In the upper part we see that toward the edges of the graphene sample charging is suppressed, whereas in the entire inner area significant charging is present. Focusing on a quite uniform area [dotted box in Figs. 3(c) and 3(d)] we find that the $\omega_{G}$ fluctuations are approximately $0.6 \mathrm{~cm}^{-1}$, which corresponds to $\Delta n \approx 2.4 \times 10^{11} \mathrm{~cm}^{-2}$. Please note that the local charge fluctuations due to electron-hole puddles presented in Ref. 19 are by one order of magnitude smaller in amplitude and have been measured at low temperature and controlled environment. In addition, our lateral resolution is limited by the laser spot size. Figs. 3(c) and 3(d), however, to AIP license or copyright; see http://apl.aip.org/apl/copyright.jsp 

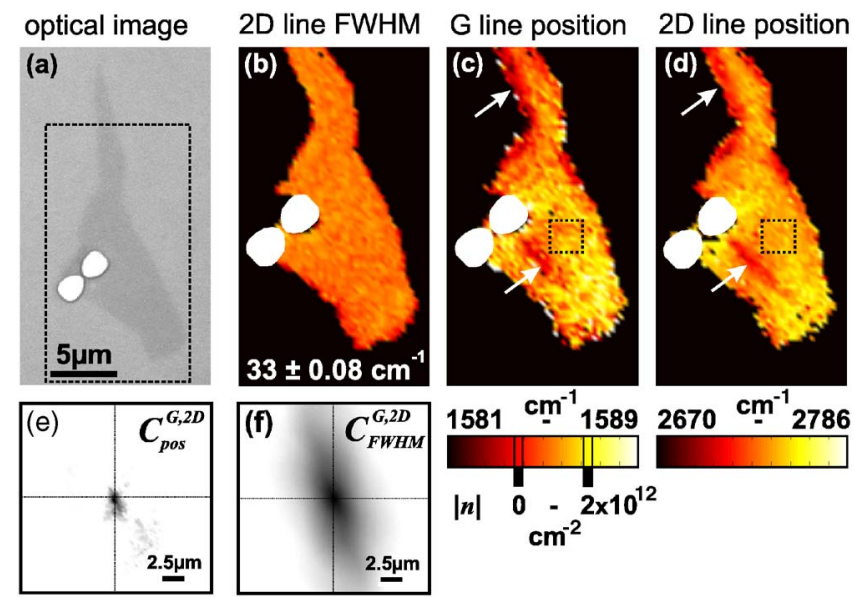

FIG. 3. (Color online) Raman images (RIs) of an isolated single-layer graphene flake on $\mathrm{SiO}_{2}$. (a) Green filtered optical image (the two white dots are metal markers). (b) RI of the FWHM of the 2D line, proving the singlelayer character of the flake. The white dashed line marks the metal marker. (c) and (d) show RIs of the G and 2D peak positions, respectively. Most attention is paid to the correlated peak fluctuations, which are due to doping fluctuations. The white arrows mark correlated low doping areas, whereas the dotted box highlights a region with low charging fluctuations. [(e) and (f)] Two-dimensional cross correlation of the fluctuations of the G and 2D line positions (widths). In both cases, the gray scale is adjusted by setting the maximum value to black. Strong correlation results (e) therefore in a centered peak at $(0,0)$, whereas low concentration leads to a distributed function [e.g., (f)].

nicely illustrate the correlation of $\mathrm{G}$ and 2D fluctuations (see, e.g., white arrows). This can be also expressed by the twodimensional cross-correlation function $C_{\omega}^{\mathrm{G}, 2 \mathrm{D}}(\Delta \mathbf{r})=\int \omega_{\mathrm{G}}(\mathbf{r}$ $+\Delta \mathbf{r}) \omega_{2 \mathrm{D}}(\mathbf{r}) d x d y$, which exhibits a clear narrow central peak [Fig. 3(e)]. In contrast, $C_{\mathrm{FWHM}}^{\mathrm{G}, 2 \mathrm{D}}(\Delta \mathbf{r})$ does show no measurable correlation of the width of $\mathrm{G}$ and 2D [Fig. 3(f)] as expected, since they are uncorrelated quantities. Indeed, the FWHM of the 2D line does not exhibit any doping dependence, as shown in Fig. 3(b). The linewidth of the 2D line stays constant at $33 \pm 0.08 \mathrm{~cm}^{-1}$ testifying its good quality for identifying single-layer graphene.

In summary, we have presented Raman shifts of the $G$ and 2D lines for back gate induced charged graphene. We have discussed the spatial variations in the $\mathrm{G}$ and $2 \mathrm{D}$ peak positions which can be attributed to different doping domains. In the low doping regime no clear distinction between electron and hole dopings can be made since for both $\mathrm{G}$ and 2D lines, stiffening is observed. However, absolute doping fluctuations can be estimated. This technique is promising to investigate, e.g., electric field distributions in (side) gated graphene devices. ${ }^{26}$

Note added. During completion of the manuscript, we became aware of a quite similar work ${ }^{27}$ in which dopingdependent fluctuations and a correlation between the position of $\mathrm{G}$ and 2D lines are observed during nonspatially resolved measurements on many different graphene flakes.

The authors wish to thank A. Bürli, S. Pisana, A. C. Ferrari, C. Roman, T. Helbling, A. Rubio, M. Lazzeri, and F. Mauri for helpful discussions. Support from the ETH FIRST Laboratory and financial support from the TH-18/03-1 grant, Swiss National Science Foundation (20021-108059/1), and NCCR nanoscience are gratefully acknowledged. L.W. acknowledges support from the French National Research Agency.

${ }^{1}$ A. K. Geim and K. S. Novoselov, Nat. Mater. 6, 183 (2007).

${ }^{2}$ M. I. Katsnelson, Mater. Today 10, 20 (2007).

${ }^{3}$ K. S. Novoselov, A. K. Geim, S. V. Morozov, D. Jiang, M. I. Katsnelson, I. V. Grigorieva, S. V. Dubonos, and A. A. Firsov, Nature (London) 438, 197 (2005).

${ }^{4}$ Y. Zhang, Y.-W. Tan, H. L. Stormer, and P. Kim, Nature (London) 438 , 201 (2005).

${ }^{5}$ Z. Chen, Y. Lin, M. Rooks, and P. Avouris, e-print arXiv:cond-mat/ 0701599.

${ }^{6}$ M. Y. Han, B. Özyilmaz, Y. Zhang, and P. Kim, Phys. Rev. Lett. 98, 206805 (2007).

${ }^{7}$ A. C. Ferrari, J. C. Meyer, V. Scardaci, C. Casiraghi, M. Lazzeri, F. Mauri, S. Piscanec, D. Jiang, K. S. Novoselov, S. Roth, and A. K. Geim, Phys. Rev. Lett. 97, 187401 (2006).

${ }^{8}$ A. Gupta, G. Chen, P. Joshi, S. Tadigadapa, and P. C. Eklund, Nano Lett. 6, 2667 (2006).

${ }^{9}$ D. Graf, F. Molitor, K. Ensslin, C. Stampfer, A. Jungen, C. Hierold, and L. Wirtz, Nano Lett. 7, 238 (2007).

${ }^{10}$ A. C. Ferrari, Solid State Commun. 143, 47 (2007).

${ }^{11}$ D. Graf, F. Molitor, K. Ensslin, C. Stampfer, A. Jungen, C. Hierold, and L. Wirtz, Solid State Commun. 143, 44 (2007).

${ }^{12}$ S. Piscanec, M. Lazzeri, F. Mauri, A. C. Ferrari, and J. Robertson, Phys. Rev. Lett. 93, 185503 (2004).

${ }^{13}$ M. Lazzeri, S. Piscanec, F. Mauri, A. C. Ferrari, and J. Robertson, Phys. Rev. B 73, 155426 (2006).

${ }^{14}$ M. Lazzeri and F. Mauri, Phys. Rev. Lett. 97, 266407 (2006).

${ }^{15}$ T. Ando, J. Phys. Soc. Jpn. 75, 124701 (2006).

${ }^{16}$ S. Pisana, M. Lazzeri, C. Casiraghi, K. S. Novoselov, A. K. Geim, A. C. Ferrari, and F. Mauri, Nat. Mater. 6, 198 (2007).

${ }^{17}$ J. Yan, Y. Zhang, P. Kim, and A. Pinczuk, Phys. Rev. Lett. 98, 166802 (2007).

${ }^{18}$ E. H. Hwang, S. Adam, and S. Das Sarma, Phys. Rev. Lett. 98, 186806 (2007).

${ }^{19}$ J. Martin, N. Akerman, G. Ulbricht, T. Lohmann, J. H. Smet, K. von Klitzing, and A. Yacoby, e-print arXiv:cond-mat/0705.2180.

${ }^{20}$ D. Graf, F. Molitor, T. Ihn, and K. Ensslin, Phys. Rev. B 75, 245429 (2007).

${ }^{21}$ C. Stampfer, A. Bürli, A. Jungen, and C. Hierold, Phys. Status Solidi B 244, 4341 (2007).

${ }^{22}$ A. Jungen, V. N. Popov, C. Stampfer, L. Durrer, S. Stoll, and C. Hierold, Phys. Rev. B 75, 041405 (2007).

${ }^{23}$ F. Molitor, D. Graf, C. Stampfer, T. Ihn, and K. Ensslin, e-print arXiv:0709.3426.

${ }^{24}$ A. Das, S. Pisana, S. Piscanec, B. Chakraborty, S. K. Saha, U. V. Waghmare, R. Yiang, H. R. Krishnamurhthy, A. K. Geim, A. C. Ferrari, and A. K. Sood, e-print arXiv:cond-mat/0709.1174.

${ }^{25} \mathrm{~L}$. Wirtz and A. Rubio (unpublished).

${ }^{26}$ F. Molitor, J. Güttinger, C. Stampfer, D. Graf, T. Ihn, and K. Ensslin, e-print arXiv:0709.2970.

${ }^{27}$ C. Casiraghi, S. Pisana, K. S. Novoselov, A. K. Geim, and A. C. Ferrari, e-print arXiv:cond-mat/0709.2566. 